\title{
Inelastic Scattering of Protons from Several Light Odd-Even Nuclei in the Energy Range from 6.0 MeV to $7.5 \mathrm{MeV}$
}

AUTHOR(S):

Kokame, Jun

\section{CITATION:}

Kokame, Jun. Inelastic Scattering of Protons from Several Light Odd-Even Nuclei in the Energy Range from 6.0 MeV to $7.5 \mathrm{MeV}$. Bulletin of the Institute for Chemical Research, Kyoto University 1963, 40(5-6): 400-400

\section{ISSUE DATE:}

1963-01-30

URL:

http://hdl.handle.net/2433/75921

RIGHT: 


\title{
ABSTRACTS
}

\section{Internal Conversion of the $1119 \mathrm{keV}$ Transition in ${ }^{65} \mathrm{Cu}$}

\author{
Sakae Shimizu and Yasuyuki Narayama \\ Nuclear Physics, 31, 600 (1962)
}

The internal $\mathrm{K}$-conversion coefficient of the $1119 \mathrm{keV}$ transition in ${ }^{65} \mathrm{Cu}$ was measured by a Siegbahn-Slätis intermediate image focussing spectrometer using the comparison method applied to the $1173 \mathrm{keV}$ gamma-rays from ${ }^{60} \mathrm{Co}$. From the known internal conversion coefficient of this transition, the value of the $1119 \mathrm{keV}$ transition was determined to be $\alpha_{\mathrm{k}}=(1.70 \pm 0.19) \times 10^{-4}$. The multipolarity assignment of the transition was also studied on the basis of the result obtained, taking into account the spin and parity assignments which have recently been made experimentally by Jambunathan et al. as well as theoretically by Lawson and Uretsky. For this transition concerned with the present work a mixture of $59 \% \mathrm{M} 1+41 \% \mathrm{E} 2$ was clearly established, corresponding to a mixing ratio $\mathrm{E} 2 /$ M 1 of 0.83 .

\section{Inelastic Scattering of Protons from Several Light Odd-Even Nuclei in the Energy Range from $6.0 \mathrm{MeV}$ to $7.5 \mathrm{MeV}$}

\section{Jun KOKAME}

Journal of the Physical Society of Japan, 16, 2101 (1961)

Protons accelerated by the $105 \mathrm{~cm}$ cyclotron of Kyoto University were used to investigate the energy dependence of the angular distributions and the reaction mechanism of the inelastically scattered protons from the odd-even nuclei, $\mathrm{B}^{11}$, $\mathrm{F}^{19}, \mathrm{Al}^{27}$ and $\mathrm{P}^{31}$ at $6.0 \sim 7.5 \mathrm{MeV}$ using conventional electronic techniques. It was found that (a) the energy dependence of the angular distribution has close relation to the excitation energy of the compound system, namely above the excitation energy of about $13 \mathrm{MeV}$ in the compound system, many patterns of the angular distributions including that of the light even-even nuclei were relatively energy-independent; (b) total cross sections were considerably smaller than that of the neighbouring even-even nuclei and less energy-dependent; (c) in the case of $\mathrm{F}^{19}$ the shapes of the angular distributions suggested the existence of a direct interaction: (d) the relative yields from the various levels of $\mathrm{Al}^{27}$ might have. some relation to its nuclear structure in the Nilsson model. 